\title{
Spacing and lag effects in free recall of pure lists
}

\author{
MICHAEL J. KAHANA \\ University of Pennsylvania, Philadelphia, Pennsylvania \\ and \\ MARC W. HOWARD \\ Syracuse University, Syracuse, New York
}

\begin{abstract}
Repeating list items leads to better recall when the repetitions are separated by several unique items than when they are presented successively; the spacing effect refers to improved recall for spaced versus successive repetition (lag $>0$ vs. lag $=0$ ); the lag effect refers to improved recall for long lags versus short lags. Previous demonstrations of the lag effect have utilized lists containing a mixture of items with varying degrees of spacing. Because differential rehearsal of items in mixed lists may exaggerate any effects of spacing, it is important to demonstrate these effects in pure lists. As in Toppino and Schneider (1999), we found an overall advantage for recall of spaced lists. We further report the first demonstration of a lag effect in pure lists, with significantly better recall for lists with widely spaced repetitions than for those with moderately spaced repetitions.
\end{abstract}

Repeating items within a list leads to an increase in the probability of their being recalled, with the beneficial effects of repetition being greatest when the repetitions are spaced (separated by other unique items). The finding of improved memory for spaced repetitions - the spacing effect - has been found in a broad range of memory tasks with many different types of study materials (Greene, 1992). In some experiments, the probability of recalling repeated items has increased monotonically with the distance, or lag, between the repetitions (Glenberg, 1976, 1977; Madigan, 1969; Melton, 1970; Underwood, 1969). The latter finding is termed the lag effect (Murdock, 1974).

A number of theoretical constructs have been proposed to account for the beneficial effects of spacing on memory for repeated items. One popular account sees subjects as devoting less attention or rehearsal to successively repeated, or massed, items (Greene, 1989; Hintzman, 1976; Rundus, 1971). This could be either a consciously controlled strategy of allocating more rehearsal to weaker items or an automatic process that responds to the novelty of a just-presented item. The possibility that rehearsal may partially explain the large spacing and lag effects typically found in free recall was supported by an early study in which the overt rehearsal technique was used (Rundus, 1971). Rundus showed that the total num-

This research was funded by National Institutes of Health Grants AG15852 and MH55687. We are grateful to Albert Huang for programming the experiment and to Joshua Jacobs for developing our Linux Experiment Programming Library. Finally, we thank Brad Wellington, Brian Kamins, and Isaac Kurtzer for help with testing the subjects. Correspondence concerning this article should be addressed to M. J. Kahana, Computational Memory Lab, Department of Psychology, University of Pennsylvania, Suite 302C, 3401 Walnut St. Philadelphia, PA 19104. ber of rehearsals was a strong predictor of later recall and that spaced items were rehearsed more than massed items. Thus, whatever process leads to the greater rehearsal of spaced items may account for some or all of the spacing effect in free recall.

A concern arises, however, due to the nature of the lists employed in studies of the spacing effect. In these studies, lists comprising a random mixture of items repeated with varying lags have typically been used. Consequently, spaced list items have inherently occupied more early list positions. At early positions, rehearsal time is distributed among the current list item and the last few items, whereas in later list positions, rehearsal time is distributed among items throughout the list (Modigliani \& Hedges, 1987; Murdock \& Metcalfe, 1978). Thus, it is possible that the higher level of recall observed for spaced items results from their having more rehearsal opportunities (Crowder, 1976).

Although many studies of the spacing effect have included additional, once-presented items at the beginning and end of the list to buffer recency and primacy effects, the primacy effect for rehearsal frequency persists well beyond the first few list positions (Brodie \& Murdock, 1977; Tan \& Ward, 2000). Even if serial position confoundings could be completely controlled, rehearsal can amplify any effects caused by the spacing of the nominal positions of the list items. Suppose, for example, that subjects attend more fully to the third repetition of a spaced item than to the third repetition of a massed item. They can use the extra time available during the presentation of the massed item to rehearse other items from the list, and these could be other massed items or spaced items. Because the distribution of rehearsals following a study item exhibits a strong recency effect over earlier presentations (Murdock \& Metcalfe, 1978), the items 
most likely to get rehearsed will be those items that are most widely dispersed throughout the list (i.e., items repeated with relatively large spacings). Thus, the interaction between the varied spacing of massed and spaced items in mixed lists and the manner in which rehearsal operates to support recall could explain both the spacing and the lag effects seen in recall tasks.

One way to eliminate the list composition account of the spacing and lag effects described above is to use a pure list design; in a pure list of massed items, displaced rehearsal from one massed item must be redistributed to some other massed item. Using a pure list design, Hall (1992) failed to find the classic advantage of spaced over massed presentation. In his critical third experiment, subjects studied two massed and two spaced lists, with words repeated twice in each list. In massed lists, items were repeated successively, whereas in spaced lists, repeated words were separated by two to four words. Hall found that in massed lists, subjects remembered more items from the first half of the list than from the second half of the list, consistent with the view that, in the latter half of the list, rehearsal time is reallocated to early list items. Despite this difference between first-half and second-half list items, overall recall was virtually identical for the two list types (59\% for both spaced and massed lists). On the basis of these results, Hall argued that the spacing effect found in mixed list experiments is a consequence of displaced rehearsal, rather than of some basic memory process.

Toppino and Schneider (1999) reexamined the issue of spacing effects in pure lists. They conducted an experiment that replicated the critical features of Hall's (1992) third experiment, but with a slightly larger range of lags for the spaced items (two to six, rather than two to four). Toppino and Schneider found a robust advantage for spaced repetition in pure lists, with $57 \%$ of the words recalled in spaced lists, as compared with only $50 \%$ recall of the words in massed lists. Massed words were better recalled in the first quarter of the list, but spaced words were better recalled at all other serial positions. Toppino and Schneider noted that in most spacing experiments, the first few and the last few items are treated as primacy and recency buffers and that, when these items are excluded from the analysis, there is a clear spacing effect throughout the remaining list positions.

Whereas Toppino and Schneider (1999) demonstrated a significant spacing effect in pure lists, the question of whether the spacing advantage increases with lag, as it does in mixed lists, remains open. Indeed, even in mixed lists, the theoretically important lag effect has not been shown in all studies (e.g., Toppino \& Gracen, 1985). As was mentioned previously, a deficiency in the processing of massed items in mixed lists could cascade into a significant lag effect due to the differential rehearsal of items with varying degrees of spacing. Similarly, a strategy of borrowing rehearsal time from spaced-long items and giving that time to spaced-short items could mask a significant lag effect. In the present study, therefore, we sought to test for the lag effect in pure lists, where only items of a single class can be rehearsed. Testing for the lag effect in pure lists also gives us an opportunity to examine spacing effects under these more stringent conditions, where displaced rehearsal cannot exaggerate the size of the spacing advantage.

To allow for larger lags, we used lists of 30 unique words, each repeated three times (as compared with Toppino \& Schneider, 1999, who used lists of 24 unique words, each presented twice). Repetitions for each list were massed, spaced short (lags 2-6), or spaced long (lags 6-20), with each subject studying lists of all three types. Three final differences between the present study and both Toppino and Schneider (1999) and Hall (1992) were that (1) words appeared at a faster rate in our study (1.5 sec, rather than $2.5 \mathrm{sec}$, per item), (2) the subjects were given a longer and more demanding distractor task following list presentation, and (3) the subjects were given more practice in the free recall task (each subject received 15 study-test lists in our study, as compared with four in Toppino \& Schneider, 1999).

\section{METHOD}

\section{Subjects}

Sixty-six Brandeis University undergraduate students were each paid $\$ 10$ for serving in a 90 -min experimental session.

\section{Procedure}

The subjects studied and attempted free recall of 15 different lists of high-frequency nouns drawn from the Toronto Noun Pool (Friendly, Franklin, Hoffman, \& Rubin, 1982). The lists consisted of 30 words, each repeated three times for a total of 90 presentations per list. List presentation was auditory, and the subjects made their responses vocally into a headset microphone. The words were presented at a rate of $1.5 \mathrm{sec}$. The screen remained blank throughout the list presentation. After list presentation, the subjects were given a distractor task involving simple arithmetic problems of the form $\mathrm{A}+\mathrm{B}+\mathrm{C}=$ ?. The subjects had to correctly answer 15 problems in a row before they could proceed to the recall phase. After completing the selfpaced distractor task (which took approximately $45 \mathrm{sec}$ ), a tone cued the subjects to recall all of the items that they could remember in any order. The vocal recall period was limited to $90 \mathrm{sec}$ for each subject. A microcomputer controlled all aspects of the experiment, including the recording of vocal responses for later scoring and analysis.

There were three list types: massed, spaced short, and spaced long. In the massed lists, each word was repeated three times successively. In the spaced-short lists, the presentation order was randomized, subject to the constraint that the lag between repetitions was at least 2 and no more than 6 . For the spaced-long lists, presentation order was randomized, subject to the constraint that interrepetition lags were at least 6 and not more than 20. We allowed for a wide range of lags (especially in the spaced-long condition) to alleviate constraints imposed by the spacing criteria that might lead to specific subsequences of items being repeated. A large range of lags also prevented the subjects from expecting to see each item once in each of the first third, second third, and final third of the spaced-long list.

Prior to the start of the experiment, the subjects were given practice at the arithmetic task. They were also informed that all the lists would include repeated items, but they were not informed about the spacing conditions. As is typical in free recall studies, we took mea- 
sures to eliminate warm-up effects by excluding the first 2 lists from our data analyses. One of these first 2 practice lists was massed, and the other was randomly chosen to be either spaced short or spaced long. Of the subsequent 12 lists, 4 were massed, 4 were spaced short, and 4 were spaced long, presented in an individually randomized order for each subject.

\section{RESULTS}

The results demonstrated the beneficial effects of spaced repetitions and of increasing lag in pure lists. Recall probability was lowest in the massed condition $(M=$ $.319, S E M=.006)$, higher in the spaced-short condition $(M=.343, S E M=.005)$, and highest in the spaced-long condition $(M=.367, S E M=.005)$. The spacing effect was substantial, with a $15 \%$ recall enhancement from the massed to the spaced-long condition. A repeated measures analysis of variance revealed a significant main effect of conditions [massed, spaced short, or spaced long; $\left.F(2,128)=12.45, M S_{\mathrm{e}}=0.003, p<.001\right]$. Planned comparisons demonstrated that both the spacing effect (the difference between the combined spaced conditions and the massed condition) and the lag effect (the difference between spaced-short and the spaced-long conditions) were statistically reliable (Bonferroni corrected $p<.001$ and $p<.01$, respectively).

Figure 1 shows the serial position curves for each of the three conditions. In the case of the spaced-short and

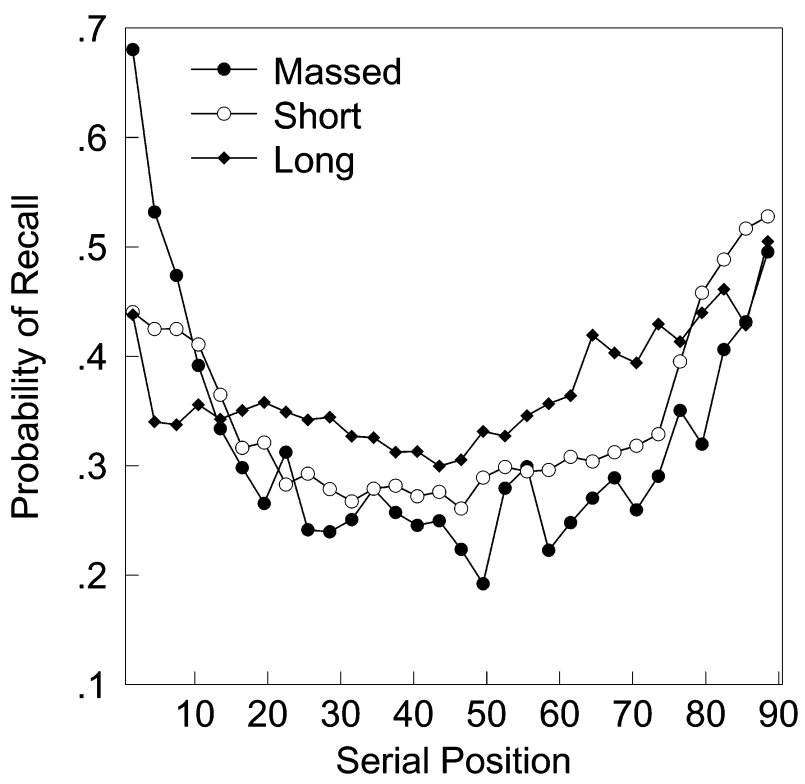

Figure 1. Probability of recall as a function of serial position for massed, spaced-short, and spaced-long conditions. Probability of recall for spaced words was calculated separately for each token. That is, if a word was presented in Positions 2, 5, and 12, recall of that word contributed to the probability of recall associated with all three serial positions. Recall probability was computed for bins of three serial positions (i.e., the first point is the average of Serial Positions 1-3, the second point is the average of Serial Positions 4-6, etc.). spaced-long conditions, one cannot calculate a standard serial position curve, because each item (token) appeared in three disparate list positions. One can, nonetheless, attempt to characterize the serial position effect by considering each occurrence of an item separately. That is, the probability of recall at serial position $i$ reflects the average recall probability for all items that had appeared in position $i$ during study.

The massed condition shows a typical serial position function with a large primacy effect and a small recency effect. The large primacy effect is typical of studies that utilize long presentation rates or encourage rehearsal (Brodie \& Murdock, 1977). The small recency effect is to be expected given the lengthy end-of-list distractor task. The spaced conditions also exhibited both primacy and recency effects, although these were probably attenuated by the fact that the items in these positions also appeared elsewhere in the list. Using this analytic approach, we find an advantage for massed items at early serial positions, in contrast to a consistent advantage for spaced items at middle and late serial positions. This primacyrecency shift in the effect of spacing was also observed when the serial position functions for spaced lists were based on either just the last occurrence of a given item (e.g., Ward, Woodward, Stevens, \& Stinson, 2003) or just the first occurrence of a given item. These serial position effects replicate Toppino and Schneider's (1999) report of an advantage for massed items in the first quartile of the list and an advantage for spaced items in the remaining quartiles.

Measuring the dependencies between successive recalls allows us to further characterize the process of recalling massed and spaced items. We know that recall of a given item will tend to follow recall of a semantically related item (e.g., Howard \& Kahana, 2002b; Pollio, Richards, \& Lucas, 1969; Romney, Brewer, \& Batchelder, 1993) or of an item studied in a nearby list position (Kahana, 1996). The latter tendency, termed the lag recency effect, illustrates the role of temporal associative factors in episodic recall (Howard \& Kahana, 1999). Given that a subject has just recalled an item from serial position $i$ and that the next response is from serial position $j$, we can plot this conditional response probability (CRP) and a function of the lag between $i$ and $j$-that is, the number of items separating $i$ and $j$ at study. These lag-CRP functions quantify the influence of encoding contiguity on later recall. ${ }^{1}$

For the massed condition, it is straightforward to calculate the lag-CRP by treating successive massed repetitions of a single word as a single presentation and computing the lag on the basis of the word serial positions, rather than the token serial position (i.e., ABSENCE ABSENCE ABSENCE HOLLOW HOLLOW HOLLOW . . . , would simply be ABSENCE HOLLOW ...). Figure 2A shows the resulting lag-CRP for the massed condition of the experiment. The lag-CRP shown in Figure 2A shows a tendency for successive recalls to come from nearby serial positions, as well as an advantage for forward over back- 

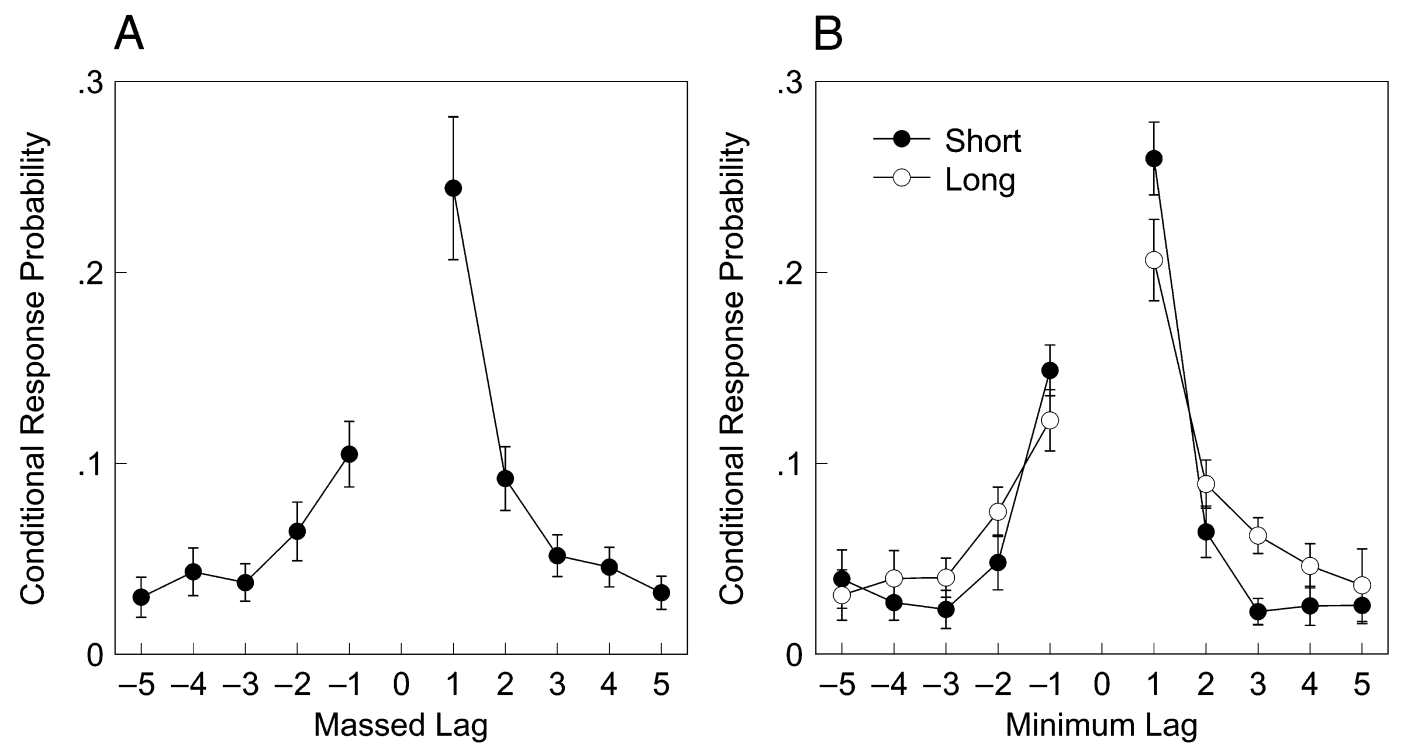

Figure 2. Conditional response probability (CRP) as a function of lag (lag-CRP) for massed, spaced-short, and spaced-long lists. In both panels, the data are collapsed across all output positions, and error bars are $95 \%$ confidence intervals calculated according to the method of Loftus and Masson (1994). (A) Lag-CRP curve for the massed condition. (B) Minimum lag-CRP curves for the spaced conditions (see the text for details).

ward recalls (associative asymmetry; see, e.g., Kahana, 2002; Kahana \& Caplan, 2002). Both of these effects replicate earlier findings for lag-CRP curves taken from lists of singly presented words (Howard \& Kahana, 1999; Kahana, 1996; Kahana, Howard, Zaromb, \& Wingfield, 2002). Apparently, temporally defined associative processes operate in massed lists in much the same way as they do in lists of single words, suggesting that repetition per se does not fundamentally alter associative processes.

For lists with spaced repetitions, such as HOLLOW PUPIL ABSENCE DARLING HOLLOW ABSENCE SAILOR . . . , the recalled pair HOLLOW-ABSENCE could be reasonably assigned lags of $+2,+1,-2$, and +5 . With three presentations of each item, as in the present study, it is possible to have nine different lags associated with any successively recalled word pair. As in the serial position analysis shown in Figure 1, it is not straightforward to compute interitem lags from lists with spaced repetitions. Nonetheless, we can use the simplifying assumption that the minimum distance between two repeated items exerts the greatest influence on recall to compute the lag-CRPs for the spaced lists. ${ }^{2}$

Figure 2B shows the results of the minimum-lag-CRP analysis for spaced lists. These curves exhibit the characteristic contiguity and asymmetry effects, with subjects successively recalling items from nearby list positions with a forward bias. In comparing the minimum-lag-CRPs for the spaced-short and the spaced-long conditions, we see that the lag-CRP curve is less sharply peaked in the spaced-long condition. This suggests that there is a broader temporal range for associations in the spaced-long condition, as compared with the spaced-short condition.

\section{DISCUSSION}

Although the spacing effect has been documented extensively in free recall, nearly all previous studies have used mixed lists - lists with massed, spaced, and oncepresented words interspersed in a random order. In mixed list studies, the finding of improved recall for spaced repetitions is vulnerable to the alternative hypothesis that subjects redistribute rehearsal time from massed to spaced items. That is, in mixed lists, subjects may use time during encoding of massed repetitions to rehearse spaced repetitions (Hall, 1992). In pure lists, in which all repetitions are either spaced or massed, such an account is untenable.

The present study compared free recall for three different list types: (1) lists whose words were each presented three times in massed fashion, (2) lists whose items were all spaced, but with short lags between the three presentations, and (3) lists whose items were spaced with long lags between the three presentations. We found that recall performance increased monotonically with the lag between the repetitions, thus demonstrating both a spacing effect (e.g., Toppino \& Schneider, 1999) and a lag effect for pure lists.

Our novel demonstration of a lag effect under these stringent conditions suggests that the lag effect is a robust feature of spacing phenomena, despite some previous failures to observe lag effects in mixed list designs (e.g., Toppino \& Gracen, 1985). The observation of a lag effect in pure lists is theoretically important because it suggests that one or more mechanisms, other than differential rehearsal, underlie the overall beneficial effects of 
repetition observed in free recall. One candidate mechanism is based on the idea of contextual variability (Estes, 1955; Glenberg, 1979). Contextual variability posits that list items are coded in relation to some type of information that changes as a function of the separation of the items. This information may reflect the operation of a contextual trace that drifts slowly over the course of item presentation (Balota, Duchek, \& Paullin, 1989; Estes, 1955; Glenberg, 1979; Howard \& Kahana, 2002a; Mensink \& Raaijmakers, 1988) or by associations among neighboring list items (Johnson, 1972; Kahana, 1996; see Figure 2). In either case, spacing the repeated items increases the number and/or the effectiveness of the retrieval cues, thus providing additional retrieval routes to the target item during recall (Crowder, 1976).

An elaborated version of the contextual variability account posits that during study, the presentation of a repeated item tends to retrieve the contextual information associated with its earlier occurrences. The reencoding of this contextual (or associative) information along with the current context results in an enriched trace (Greene, 1989; Howard \& Kahana, 2002a). Not only do the multiple occurrences of an item have different contexts, thus affording multiple retrieval routes, but also the context of earlier occurrences, along with the new context, becomes associated with later occurrences of an item. Greene (1989) noted several lines of evidence consistent with the view that study phase retrieval provides the best account of spacing effects in free recall. Among these sources of evidence is the finding that spaced presentations can actually produce higher levels of recall than would be expected if the items were encoded independently (Ross \& Landauer, 1978).

Raaijmakers (2003) examined how well a version of the search of associative memory (SAM) retrieval model (Raaijmakers \& Shiffrin, 1980, 1981) could account for spacing effects in paired-associate tasks. Raaijmakers' implementation of SAM incorporated mechanisms for contextual fluctuation (as in Mensink \& Raaijmakers, 1988) and study phase retrieval. Using these mechanisms, SAM could explain a number of key features of spacing effects in paired-associate tasks.

Although contextual retrieval theories have been able to account for many of the spacing phenomena observed in mixed list experiments, they also make clear predictions about the existence of robust spacing and lag effects in pure lists. These predictions are strongly supported by the present findings. It is of course possible that the present findings reflect a phenomenon specific to free recall that does not generalize to cued memory tasks. It will be important, in future work, to determine whether there is some point at which the lag effect reverses, with very long inter-repetition lags resulting in worse subsequent memory for repeated items (as compared with moderate lags). This issue of the monotonicity of the lag effect in free recall may prove critical in testing the study phase retrieval version of contextual variability theory.

\section{REFERENCES}

Balota, D. A., DucheK, J. M., \& Paullin, R. (1989). Age-related differences in the impact of spacing, lag, and retention interval. Psychology \& Aging, 4, 3-9.

BRODIE, D. A., \& MURDOCK, B. B. (1977). Effects of presentation time on nominal and functional serial position curves in free recall. Journal of Verbal Learning \& Verbal Behavior, 16, 185-200.

Crowder, R. G. (1976). Principles of learning and memory. Hillsdale, NJ: Erlbaum.

Estes, W. K. (1955). Statistical theory of distributional phenomena in learning. Psychological Review, 62, 369-377.

Friendly, M., Franklin, P. E., Hoffman, D., \& Rubin, D. C. (1982). The Toronto Word Pool: Norms for imagery, concreteness, orthographic variables, and grammatical usage for 1,080 words. Behavior Research Methods \& Instrumentation, 14, 375-399.

Glenberg, A. M. (1976). Monotonic and nonmonotonic lag effects in paired-associate and recognition memory paradigms. Journal of Verbal Learning \& Verbal Behavior, 15, 1-16.

GLENBERG, A. M. (1977). Influences of retrieval processes on the spacing effect in free recall. Journal of Experimental Psychology: Human Learning \& Memory, 3, 282-294.

Glenberg, A. M. (1979). Component-levels theory of the effects of spacing of repetitions on recall and recognition. Memory \& Cognition, 7, 95-112.

GREENE, R. L. (1989). Spacing effects in memory: Evidence for a twoprocess account. Journal of Experimental Psychology: Learning, Memory, \& Cognition, 15, 371-377.

GreEne, R. L. (1992). Human memory: Paradigms and paradoxes. Hillsdale, NJ: Erlbaum.

HALL, J. W. (1992). Unmixing effects of spacing on free recall. Journal of Experimental Psychology: Learning, Memory, \& Cognition, 18, 608-614.

Hintzman, D. L. (1976). Repetition and memory. In G. H. Bower (Ed.), The psychology of learning and memory (pp. 47-91). New York: Academic Press.

Howard, M. W., \& KahanA, M. J. (1999). Contextual variability and serial position effects in free recall. Journal of Experimental Psychology: Learning, Memory, \& Cognition, 25, 923-941.

HowARD, M. W., \& KaHANA, M. J. (2002a). A distributed representation of temporal context. Journal of Mathematical Psychology, 46, 269-299.

Howard, M. W., \& KAHANA, M. J. (2002b). When does semantic similarity help episodic retrieval? Journal of Memory \& Language, $\underline{\mathbf{4 6}}_{2}$ 85-98.

JoHNSON, M. K. (1972). Organizational units in free recall as a source of transfer. Journal of Experimental Psychology, 94, 300-307.

Kahana, M. J. (1996). Associative retrieval processes in free recall. Memory \& Cognition, 24, 103-109.

KAHANA, M. J. (2002). Associative symmetry and memory theory. Memory \& Cognition, 30, 823-840.

KaHANA, M. J., \& CAPLAN, J. B. (2002). Associative asymmetry in probed recall of serial lists. Memory \& Cognition, 30, 841-849.

Kahana, M. J., Howard, M. W., Zaromb, F., \& WingField, A. (2002). Age dissociates recency and lag-recency effects in free recall. Journal of Experimental Psychology: Learning, Memory, \& Cognition, 28, 530-540.

LoFTUS, G. R., \& MAsson, M. E. J. (1994). Using confidence intervals in within-subject designs. Psychonomic Bulletin \& Review, 1, 476-490.

Madigan, S. A. (1969). Intraserial repetition and coding processes in free recall. Journal of Verbal Learning \& Verbal Behavior, 8, 828835.

Melton, A. W. (1970). The situation with respect to the spacing of repetitions and memory. Journal of Verbal Learning \& Verbal Behavior, 9. 596-606.

Mensink, G.-J. M., \& RaAijmakers, J. G. W. (1988). A model for interference and forgetting. Psychological Review, 95, 434-455.

Modigliani, V., \& Hedges, D. G. (1987). Distributed rehearsals and the primacy effect in single-trial free recall. Journal of Experimental Psychology: Learning, Memory, \& Cognition, 13, 426-436. 
Murdock, B. B. (1974). Human memory: Theory and data. Potomac, MD: Erlbaum.

Murdock, B. B., \& Metcalfe, J. (1978). Controlled rehearsal in single-trial free recall. Journal of Verbal Learning \& Verbal Behavior, 17, 309-324.

Pollio, H. R., Richards, S., \& Lucas, R. (1969). Temporal properties of category recall. Journal of Verbal Learning \& Verbal Behavior, $\mathbf{8}_{2}$ $\underline{529-536 .}$

RAAIJMAKERS, J. G. W. (2003). Spacing and repetition effects in human memory: Application of the SAM model. Cognitive Science, 27, 431452.

RaAiJmakers, J. G. W., \& Shiffrin, R. M. (1980). SAM: A theory of probabilistic search of associative memory. In G. H. Bower (Ed.), The psychology of learning and motivation: Advances in research and theory (Vol. 14, pp. 207-262). New York: Academic Press.

RaAiJmakers, J. G. W., \& Shiffrin, R. M. (1981). Search of associative memory. Psychological Review, 88, 93-134.

Romney, A. K., Brewer, D. D., \& BATCHELder, W. H. (1993). Predicting clustering from semantic structure. Psychological Science, $\mathbf{4}$, 28-34.

Ross, B., \& LANDAUER, T. (1978). Memory for at least one of two items: Test and failure of several theories of spacing effects. Journal of Verbal Learning \& Verbal Behavior, 17, 669-680.

Rundus, D. (1971). An analysis of rehearsal processes in free recall. Journal of Experimental Psychology, 89, 63-77.

TAN, L., \& WARD, G. (2000). A recency-based account of the primacy effect in free recall. Journal of Experimental Psychology: Learning, Memory, \& Cognition, 26, 1589-1626.

Toppino, T. C., \& GracEN, T. F. (1985). The lag effect and differential organization theory: Nine failures to replicate. Journal of Experimental Psychology: Learning, Memory, \& Cognition, 11, 185-191.

TopPINO, T. C., \& SchneIDER, M. A. (1999). Observation: The mix-up regarding mixed and unmixed lists in spacing-effect research. Journal of Experimental Psychology: Learning, Memory, \& Cognition, 25, 1071-1076.
UNDERWOOD, B. J. (1969). Some correlates of item repetition in freerecall learning. Journal of Verbal Learning \& Verbal Behavior, 8, 8394.

Ward, G., Woodward, G., Stevens, A., \& Stinson, C. (2003). Using overt rehearsals to explain word frequency effects in free recall. Journal of Experimental Psychology: Learning, Memory, \& Cognition, 29, 186-210.

\section{NOTES}

1. One calculates the lag-CRP by tallying the number of times a transition of a certain lag, $x$, is made and then counting the number of times that a transition of lag $x$ could have been made within a given trial. Summing over all the trials for a given subject, the lag-CRP function shows the number of times a transition of lag $x$ was made divided by the number of times that a transition of lag $x$ could have been made.

2. A complicating factor in this analysis is that there may be multiple potential recalls at a given minimum lag and that given minimum lag may be both recalled and unrecalled. According to the method described above, for instance, we would assign a lag of +1 to recalling ABSENCE after HOLLOW. However, the transition HOLLOW-PUPIL, which was not recalled, would also be assigned a lag of +1 . The lag +1 could be considered to be both recalled and not recalled. This seems unfair in some sense- +1 was recalled as often as it could have been. To try to obtain a cleaner measure of the effect of lag, the CRP for a given minimum lag was updated no more than once for a given trial, and the recalled lag was updated only as a positive response. In the special case in which a pair of words appeared at a lag of $+N$ and $-N$, we counted $+N$ as the shorter distance, in acknowledgment of the widespread finding of a forward-biased asymmetry in associative processes (Kahana, 1996).

(Manuscript received May 15, 2003; revision accepted for publication February 16, 2004.) 